\title{
Flexicorticotomía. Una técnica quirúrgica para tratamiento de ortodoncia. Reporte de un caso
}

\section{Ridge split. A surgical technique for orthodontic treatment. A case report}

\author{
Díaz Caballero A*, Herrera Barrios F**, Herrera Herrera A***
}

\section{RESUMEN}

El tratamiento ortodóncico es un procedimiento que se utiliza para la mejoría de las anomalías de forma, posición, relación y función de las estructuras dentomaxilofaciales, mediante el uso y control de diferentes tipos de fuerzas, observándose principalmente en niños menores de 12 años.

La flexicorticotomía es un procedimiento quirúrgico que se utiliza principalmente para la colocación de implantes en aquellas personas que presentan disminución de la cortical, pero también se puede tomar como una buena técnica coadyuvante para facilitar el tratamiento ortodóncico en pacientes mayores en el cual el movimiento dentario es más lento y hay una mayor condensación ósea impidiendo que se puedan posicionar. Es decir, cuando no se produce movimiento por la reducción en la eficacia de los tejidos.

El objetivo del presente artículo es mostrar el excelente uso de la técnica de flexicorticotomía como ayuda clínica en los tratamientos ortodónticos en adultos, por medio de un caso clínico que tuvo un adecuado resultado, sin ninguna complicación.

Palabras clave: Ortodoncia correctiva, aumento de la cresta alveolar, expansión de tejido, procesos alveolares, procedimientos quirúrgicos ambulatorios.

\section{SUMMARY}

Orthodontic treatment is a procedure that is used for the improvement of abnormal shape, position, relation and function of structures dentomaxilofaciales, using and controlling different types of forces, mainly observed in children younger than 12 years.

The ridge split is a surgical procedure that is used primarily for the placement of dental implants in those people who fall in cortical, but also can be taken as a good adjunct technique to facilitate orthodontic treatment in patients with older age at which the tooth movement is slower and there is less bone preventing condensation which teeth can be positioned. When no movement is produced by the reduction in the effectiveness of the tissues.

The aim of this paper is to propose a technique to help ridge split as and adjunct in clinical orthodontic treatment in adults, through a case presentation that had a good result without any complication..

Key words: Orthodontics corrective, alveolar ridge augmentation, tissue expansión, alveolar process, ambulatory surgical procedures.

Fecha de recepción: 11 de junio de 2009.

Aceptado para publicación: 22 de junio de 2009.

* Odontólogo, Universidad de Cartagena. Especialista en Periodoncia. Docente titular Univ. de Cartagena.

** Odontólogo, Universidad de Cartagena. Cirujano Maxilofacial Universidad de Buenos Aires. Docente de Cátedra de pregrado y de postgrado Universidad de Cartagena.

*** Estudiante VII semestre Facultad de Odontología Universidad de Cartagena.

Díaz Caballero A, Herrera Barrios F, Herrera Herrera A. Flexicorticotomía. Una técnica quirúrgica para tratamiento de ortodoncia. Reporte de un caso. Av. Odontoestomatol 2010; 26 (5): 243-247. 


\section{INTRODUCCIÓN}

En el intento de mejorar las maloclusiones que siguen siendo validas en la actualidad, la ortodoncia nació como especialidad en 1906 cuando Edward Angle, en Estados Unidos, sienta las bases del diagnostico y tratamiento dirigidas hacia unos objetivos funcionales, estéticos y psicosociales (1).

La ortodoncia basa sus objetivos en la corrección ósea y dental, de las estructuras faciales, tendiendo a conseguir aquellas normas estéticas y funcionales, que deciden los padres, los pacientes y el profesional, con su mejor criterio, para rehabilitar social y psicológicamente al paciente $(2,3)$.

Los aparatos ortodóncicos se adaptan a cualquier boca, aunque la edad ideal para realizar el tratamiento es aquella en la que el hueso y los tejidos que sostienen el diente en su sitio están en fase de desarrollo o crecimiento, lo que ocurre, aproximadamente, hasta los catorce años (4). A partir de ahí $y$, principalmente, en la época adulta, la adaptación de los dientes a los cambios que se provocan artificialmente es más complicada, pero no imposible (5).

Estos tratamientos ortopédicos dentomaxilofaciales, tienen por objeto prevenir y corregir las malas posiciones dentarias y las deformidades maxilofaciales durante el curso del crecimiento, pero en los adultos debe tomarse la precaución de controlar el estado de salud periodontal, pues en casos de destrucciones avanzadas del hueso alveolar y, por consiguiente, de la membrana periodontal, el diente tendrá dificultades para afianzarse una vez terminado el movimiento, por ausencia o disminución de oposición ósea (6).

Como se sabe, los movimientos ortodóncicos tienen lugar en tejidos vivos, de modo que cualquier descuido en la aplicación del aparato mecánico tiende a dañarlos y a provocar reabsorciones radiculares, necrosis, alteraciones gingivales y un sinnúmero de problemas deriva dos del uso de presiones exageradas; por tanto, parece existir una relación directamente proporcional entre la cantidad de fuerza aplicada sobre el diente y la cantidad y cualidad de las reabsorciones producidas sobre él (6).
Es por éste que en 1992 Simion y col. presentó la propuesta de la división de la cresta ósea y la manipulación de los huesos. En el cual el objetivo era crear un espacio de forma longitudinal en los pacientes con atrofias en el maxilar, dividiendo la parte superior de la cresta ósea en dos con el fin de colocar los implantes en el espacio creado (7).

La flexicorticotomía fue descrita por Bruschi y Scipioni en el año 1990 después de buenos y predecibles resultados con el propósito de desarrollar una nueva técnica quirúrgica en el tratamiento del edentulismo atrófico (8). También en aquellos pacientes adultos jóvenes para corregir defectos alveolares producidos por traumatismos o por agenesias de uno o más dientes y que luego su uso se amplió en todas las maniobras quirúrgicas que pretendan llegar al hueso esponjoso para sacar partido de la mayor elasticidad de este sobre el hueso cortical como es en el caso de la necesidad de ensanchamiento previo a la colocación de un implante o en las maniobras de la preparación de una osteodistracción (9).

Se utiliza en distintas situaciones clínico/quirúrgicas en la cual se realiza la separación de las corticales vestibular y lingual/palatina de la apófisis alveolar en aquellos casos en los que no se dispone el grosor alveolar adecuado para colocar implantes y conseguir una estabilidad primaria $(8,10)$.

\section{CASO CLÍNICO}

Paciente masculino de 34 años de edad, que acudió a consulta odontológica para la realización de tratamiento ortodóntico por presencia de diastemas anteroinferiores desde hace dos años; respecto a sus antecedentes médicos no reportó datos de relevancia alguna.

Después de un año y medio del tratamiento ortodóntico el diastema entre el canino y el central no cerraba el espacio con la aplicación de las fuerzas (Fig. 1). Se tomaron radiografías y se observó el aumento de la condensación del hueso y disminución de la cresta ósea, siendo esta tan delgada que no permitiría el movimiento dentario solo con las fuerzas ortodóncicas. Diagnóstico de un aumento 


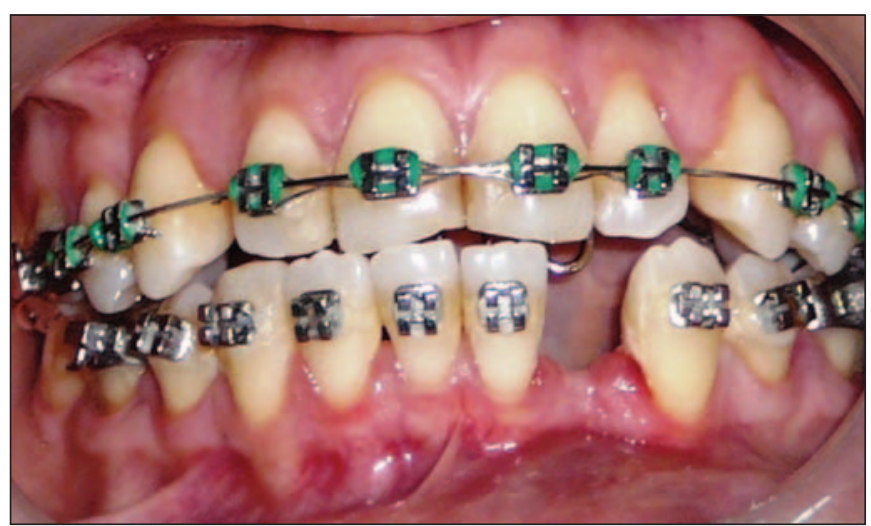

Fig. 1. Presencia de diastema entre el central y el canino inferior.

de la condensación ósea. El plan de tratamiento a seguir fue la realización de una flexicorticotomía y la colocación de un relleno óseo.

Teniendo en cuenta que el requisito mínimo para realizar una flexicorticotomía es que las paredes de la cortical tengan $1 \mathrm{~mm}$ de espesor al menos (11). Se encontró que en el paciente fue $2,3 \mathrm{~mm}$; haciendo posible la realización del procedimiento y colocación del relleno con injerto óseo.

Una vez tomada la radiografía días antes de la cirugía, se realizó una incisión crestal y la eliminación del periostio con elevación del colgajo hasta tener la cresta ósea al descubierto, se observa una depresión marcada que no permite la posibilidad de mover un diente en esa parte de hueso tan estrecho (Fig. 2). Luego se realizó una fractura controlada con una expansión debido a la elasticidad de la cortical con martillo y cincel hasta obtener un surco de 0,3 mm de anchura y $5 \mathrm{~mm}$ de diámetro de profundidad permitiendo la separación de ambas tablas sin rotura (Fig. 3).

Se colocó relleno con injerto óseo autólogo, desecado, desantigenizado, liofilizado, obtenido en banco de tejidos humanos, facilitando que el coagulo encuentre un biomaterial que ayudar a formar hueso en esa zona y a su vez ayuda a mantener la separación de las corticales. Finalmente se suturó el plano vestibular y palatino para impedir tensiones en el tejido blando, con seda 4-0. Se prescribió amoxicilina de $500 \mathrm{mg}$, cápsula por 7 días y acetaminofen de $500 \mathrm{mg}$ tabletas por 5 días en presencia de dolor. Al

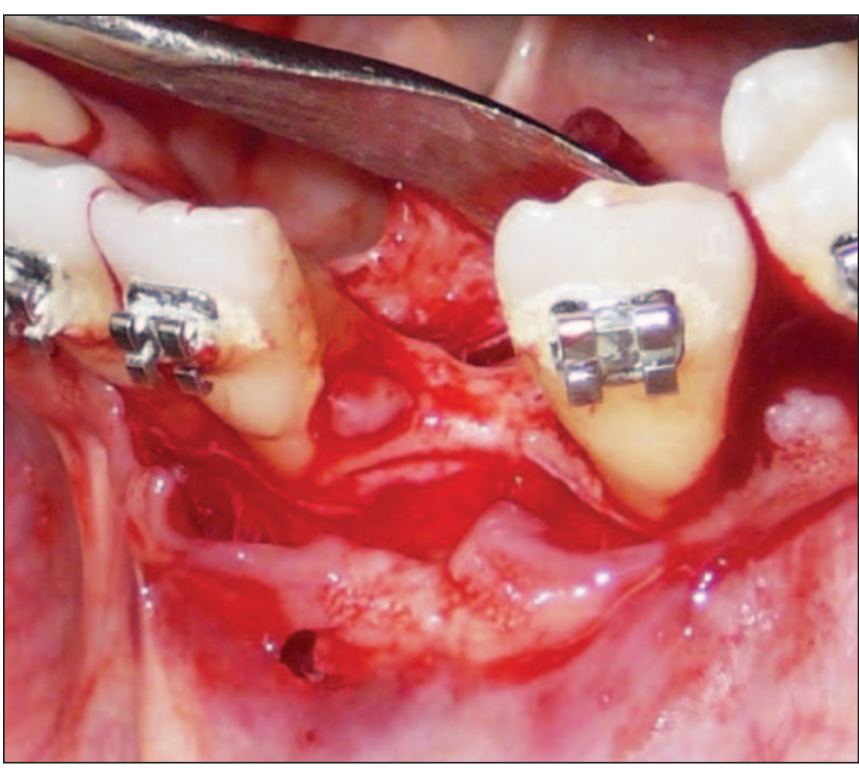

Fig. 2. Se observa la cresta ósea al descubierto la cual esta bastante disminuida, con una depresión marcada que no permite la posibilidad de correr el diente.

octavo día se observa nuevamente la evolución del paciente con excelente mejora optando por retirar los puntos.

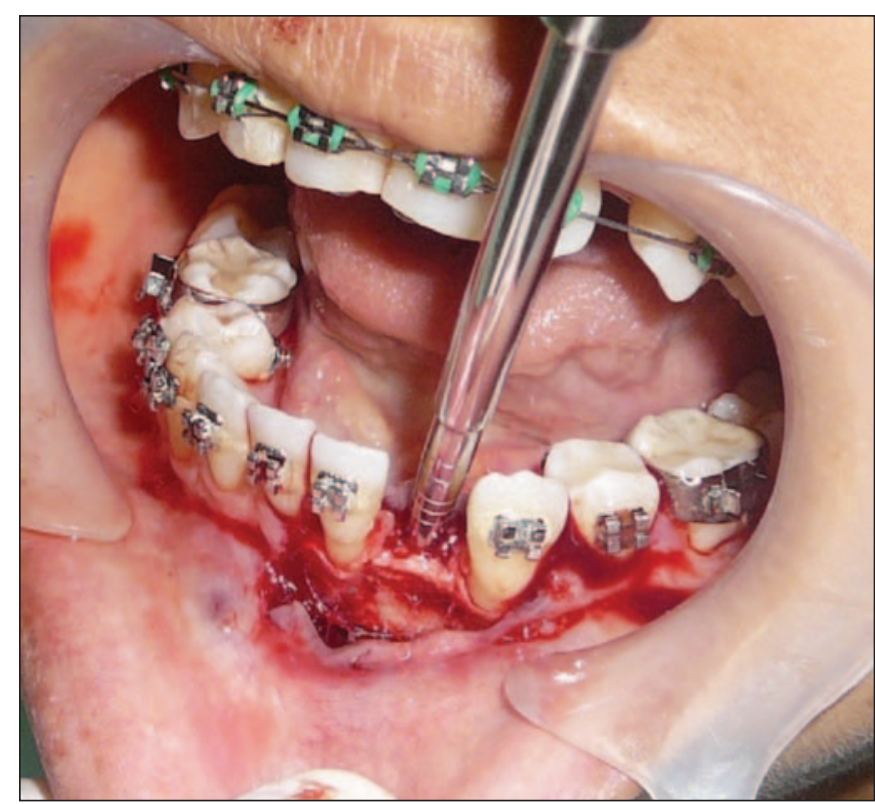

Fig. 3. Separación de las tablas óseas con el cincel y martillo, en el sitio de contacto con el hueso, dando la apariencia de un principio de reabsorción por la intensidad de la fuerza y la resistencia del hueso ante el movimiento. 
Tres meses después se observó clínicamente que el diastema había desaparecido; con la radiografía periapical final se observó regeneración ósea positiva y disminución en el espacio entre el central y el canino (Fig. 4).

\section{DISCUSIÓN}

La mayoría de autores utilizan la técnica de la flexicorticotomía como una técnica quirúrgica usada en los casos de pacientes edéntulos que requieran un implante, cuya reabsorción se ve bastante marcada y buscan crear un espacio en la cresta ósea para la colocación de estos mismos (12).

Además de que es un proceso excelente para ganar espacio en la colocación de implantes. En el presente caso, se hace hincapié en utilizar la flexicorticotomía no solo con este fin sino como ayuda para aquellos pacientes en que la condensación ósea esté aumentada e impidan el tratamiento ortodóntico por la formación de cuellos de botella (13).

El uso de este procedimiento ofrece varias ventajas dependiendo de la finalidad de la misma además de la expansión de la cresta ósea en sentido horizontal para la colocación de implantes, la utilización de corticotomías permite desplazar las corticales consiguiendo sustrato óseo suficiente para el alojamiento de los implantes (14).

El defecto expandido se cura de manera similar a una cavidad de extracción. En ciertos casos, los

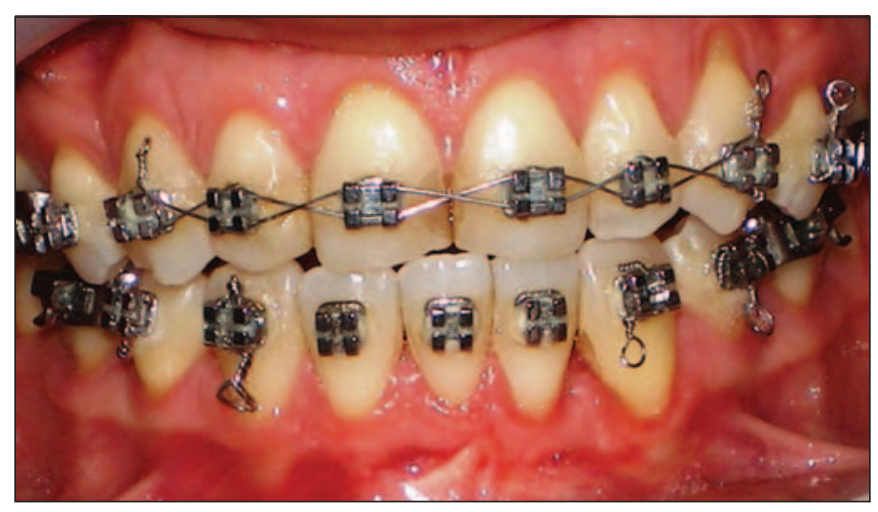

Fig. 4. Resultados después de 3 meses del procedimiento, en el cual se observa el cierre del espacio que existía entre el canino y el central, con excelentes resultados. pacientes pueden usar sus dentaduras luego de la operación. Los lugares quirúrgicos secundarios no son un prerrequisito y se puede lograr la colocación simultánea del implante durante la expansión de la cresta. La limitación de esta técnica radica en una imposibilidad de crear hueso verticalmente (15).

En 1992 Simion, Baldon, Zaffe (16) demostraron con estudios biométricos que por medio de la flexicorticotomía se consiguen expansiones entre 1 y 4 mm y que al examen histológico se puede observar una regeneración del tejido óseo entre las dos partes de la división de la cresta. Lo que significa que es una buena técnica a recomendar por la poca morbilidad y excelentes resultados.

También permite corregir los defectos anatómicos vestibulares a modo de depresión que suelen acompañar a la cresta estrecha. Suele realizarse en estos casos con discos circulares montador en motores de baja velocidad o turbina (14).

En ortodoncia para lograr una expansión palatina rápida en la que: la expansión rápida de paladar asistida quirúrgicamente es una de las opciones terapéuticas de los trastornos transversales del maxilar superior, consistiendo en una corticotomía de la pared lateral del maxilar combinada con una osteotomía palatina media transincisal (17).

De esto se deduce que una terapéutica ortodóncica sistemática, luego del acto quirúrgico, proporciona un rápido movimiento y la afirmación de los dientes reubicados, observando notables propiedades reparadoras y la versatilidad del tejido conjuntivo abarcado (17).

\section{BIBLIOGRAFÍA}

1. Cunningham SJ, Jones SP, Hodges SJ, Horrocks EN, Hunt NP, Moseley HC, Noar JH. Advances in orthodontics. Prim Dent Care. 2002 Jan;9(1):5-8.

2. Scott P, DiBiase AT, Sherriff $M$, Cobourne MT. Alignment efficiency of Damon 3 self-ligating and conventional orthodontic bracket systems: a randomized clinical trial. Am J Orthod Dentofacial Orthop. 2008 Oct;134(4):470.e1-8. 
3. Day PF, Kindelan SA, Spencer JR, Kindelan JD, Duggal MS. Dental trauma: part 2. Managing poor prognosis anterior teeth-treatment options for the subsequent space in a growing patient. $\mathrm{J}$ Orthod 2008 Sep;35(3):143-55.

4. Kolawole KA, Otuyemi OD, Jeboda SO, Umweni AA. The need for orthodontic treatment in a school and referred population of Nigeria using the index of orthodontic treatment need (IOTN). Odontostomatol Trop 2008 Jun;31(122):11-9.

5. Chaushu S, Becker A, Chaushu G. Lingual orthodontic treatment and absolute anchorage to correct an impacted maxillary canine in an adult. Am J Orthod Dentofacial Orthop 2008 Dec;134(6):811-9.

6. Generson RM. Combined surgical and orthodontic management of anterior open biten using corticotomy. J Oral Surg 1978;36(3): 216-9.

7. Simion M, Saldoni M, Zaffe D. Jawbone enlargement using immediate implant placement associated with a split crest technique and guided tissue regeneration. Int $\mathrm{J}$ Periodontics Restorative Dent 1992;2:462-473.

8. Scipioni A, Bruschi GB, Calesini G, Bruschi E, De Martino C. Bone regeneration in the edentulous ridge expansion technique: histologic and ultrastructural study of 20 clinical cases. Int J Periodontics Restorative Dent 1999 Jun;19(3): 269-77.

9. Lee JK, Chung KR, Baek SH. Treatment outcomes of orthodontic treatment, corticotomyassisted orthodontic treatment, and anterior segmental osteotomy for bimaxillary dentoalveolar protrusion. Plast Reconstr Surg 2007 Sep 15; 120(4):1027-36.

10. Elian N, Jalbout Z, Ehrlich B, Classi A, Cho SC, Al-Kahtani F, Froum S, Tarnow DP. A two-stage full-arch ridge expansion technique: review of the literature and clinical guidelines. Implant Dent 2008 Mar;17(1):16-23.

11. Veltri $M$, Ferrari $M$, Balleri P. One-year outcome of narrow diameter blasted implants for rehabilitation of maxillas with knife-edge resorption. Clin Oral Implants Res 2008 Oct;19 (10):1069-73.

12. Enislidis G, Wittwer G, Ewers R. Preliminary report on a staged ridge splitting technique for implant placement in the mandible: a technical note. Int J Oral Maxillofac Implants 2006;21(3):445-9.

13. Oikarinen KS, Sàndor GK, Kainulainen VT, Salonen-Kemppi M. Augmentation of the narrow traumatized anterior alveolar ridge to facilitate dental implant placement. Dent Traumatol 2003;19(1):19-29.

14. Aghaloo TL, Moy PK. Which hard tissue augmentation techniques are the most successful in furnishing bony support for implant placement?. Int $\mathrm{J}$ Oral Maxillofac Implants 2007;22 Suppl:49-70.

15. Sethy A, Kaus T. Maxillary ridge expansion with simultaneous implant placement: 5-year results of an ongoing clinical study. Int $\mathrm{J}$ Oral Maxillofac Implants 2000;15:491-499.

16. Simion M, Baldoni M, Zaffe D. Jawbone enlargement using immediate implant placement associated with a split-crest technique and guided tissue regeneration. Int $\mathrm{J}$ Periodontics Restorative Dent 1992;12(6):462-73.

17. Bernstein S, Cooke J, Fotek P, Wang HL. Vertical bone augmentation: where are we now?. Implant Dent 2006 Sep;15(3):219-28.

\section{CORRESPONDENCIA}

Antonio Díaz Caballero.

Facultad de Odontología

Correo electrónico: alejandrah03@gmail.com. 